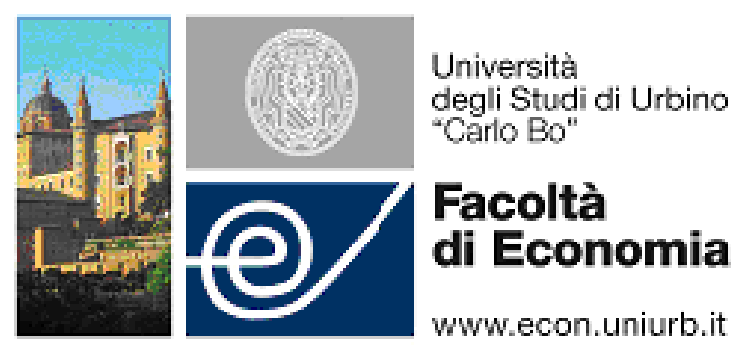

WP-EMS

Working Papers Series in Economics, Mathematics and Statistics

"Cournot Duopoly when the Competitors Operate Multiple Production Plants"

- Fabio Tramontana, (U. Ancona \& U. Urbino)

- Laura Gardini, (U. Urbino)

- Tönu Puu (Umeå University, Sweden) 


\title{
Cournot Duopoly when the Competitors operate Multiple Production Plants
}

\author{
${ }^{1}$ Fabio Tramontana, ${ }^{2}$ Laura Gardini, ${ }^{3}$ Tönu Puu \\ ${ }^{1}$ Department of Economics, Ancona University, Italy \\ ${ }^{2}$ Department of Economics and Quantitative Methods, Urbino University, Italy \\ ${ }^{3}$ CERUM, Umeå University, SE-90187 Umeå, Sweden
}

\begin{abstract}
This article considers a Cournot duopoly under an isoelastic demand function and cost functions with built-in capacity limits. The special feature is that each firm is assumed to operate multiple plants, which can be run alone or in combination. Each firm has two plants with different capacity limits, so each has three cost options, the third being to run both plants, dividing the load according to the principle of equal marginal costs. As a consequence, the marginal costs functions come in three disjoint pieces, so the reaction functions, derived on basis of global profit maximization, may also consist of disjoint pieces. This is reflected in a particular bifurcation structure, due to border collision bifurcations, and to particular basin boundaries, related to the discontinuities. It is shown that stable cycles may coexist, and the non-existence of unstable cycles constitutes a new property. We also compare the coexistent short periodic solutions in terms of the resulting real profits.
\end{abstract}

JEL classification: C15, C62, D24, D43

\section{Introduction}

The present paper $^{1}$ contributes to the analysis of Cournot oligopoly models where each of the competitors has the option of utilizing several plants. Economists have been interested in oligopoly models since many years. After the dynamic adjustment process proposed by Cournot (1838), a linear model always converging to a unique equilibrium (later called Cournot Equilibrium), several contributions followed to show that the Cournot equilibrium may be unstable (see e.g. Palander 1939, Theocharis 1960, Fisher 1961, McManus and Quandt 1961) or not unique (Robinson 1933, Palander 1936,1939). The literature dealing with Cournot models has increased greatly in the last two decades: The existence and stability of Cournot equilibria have been analyzed by Furth

${ }^{1}$ The authors are grateful to anonymous referees for their useful suggestions and remarks, which allowed to improve our work. 
(1986), via a cubic marginal costs curve, Bonanno (1988), via a cubic marginal revenues curve, and local/global stability properties became important issues.

A rich literature stream on this subject is related to duopoly or triopoly models, usually described by continuous and smooth reaction functions (see e.g. Okuguchi 1976, Al-Nowaihi and Levine 1985, Dana and Montrucchio 1986, Puu 1991, 1996, Kopel 1996). A peculiarity of these models is the existence of multistability. This means that several attracting sets coexist, and the long run behaviour of the game may give rise to different alternatives (see e.g. Agiza 1999, Agiza et al. 1999, Bischi and Naimzada, 1999, Bischi et al. 2000, Bischi and Kopel, 2001, Puu and Sushko 2002). In these models the local bifurcations (changes in the stability of the equilibria or the cycles) are quite standard and well known, being related to smooth changes of the eigenvalues of the linear approximation of the dynamic model. Moreover, global properties (i.e. not related to the eigenvalues) are used to investigate the shape and qualitative changes of the basins of attraction. In these models the basins are bounded by the stable sets of saddle points or unstable equilibria.

Besides these smooth models, some authors proposed duopoly games characterized by piecewise smooth or even discontinuous reaction functions. In these cases, the dynamic analysis may involve the occurrence of so-called bordercollision bifurcations, which are related to the crossing of the equilibria or cycles points through sets where the dynamical system is not differentiable, that separate regions where the maps that represent the dynamical system are differentiable but different. These bifurcations may cause sudden stability switches and/or the appearance/disappearance of cycles. The study of border-collision bifurcation is not new in models applied to economics (see e.g. the pioneering works by Hommes 1991, Hommes and Nusse 1991, Hommes 1995, Hommes et al. 1995). The applications of such techniques has been used in business cycle models and in oligopoly models for a few years (see e.g. Gallegati et al. 2003, Puu et al. 2002, 2005, Sushko et al. 2003, Puu and Sushko 2006). It is worth mentioning that the main results on this subject are due to Nusse and Yorke 1992, 1995, Maistrenko et al., 1993, 1995, 1998, Di Bernardo et al. 1999, Banerjee et al. 2000a;b, Halse et al. 2003, Zhanybai et al. 2003, and applications to economics also, as in Sushko et al. 2005, 2006.

Cournot duopoly models with unstable equilibria and discontinuities were already introduced by Furth (1986) and even before by Palander (1939), who also proposed several situations where multistability could occur. For example, a case with a piecewise linear Robinson-type demand function, where the elasticity increased drastically when price was lowered. Then the marginal revenue function become discontinuous with jumps, and the corresponding reaction function is discontinuous as well. Moreover, the reaction functions could intersect in several points, Cournot (or Nash) equilibria, giving rise to multistability. This case was reconsidered in detail by two of the present authors (see Puu et al. 2002).

Palander also developed a case where each firm could operate several production plants, some suitable for small scale production (low fixed costs, high marginal costs), other suitable for large scale production (high fitting up costs, 
low marginal costs). In this case it was the marginal cost function that was discontinuous, and, again, multistability could occur. To our knowledge, this model was never further studied. Like in the kinked demand case, Palander based his study on linear functions. However, we prefer to skip the linear format, using an isoelastic demand function, and non-linear cost functions with built in capacity limits.

In our model we suppose that each firms has two plants. However, as the firms can either operate each of the plants separately, or both in combination dividing production between the plants according to the principle of equal marginal costs, it follows that the firms actually have three cost options. Typically, the option chosen depends on output. Suppose we can classify the plants according to their optimal scale of operation; then at a small output the small scale plant will be chosen, with increasing output the choice will shift to the plant appropriate for larger scale production, and eventually both plants will be taken in use, the combination representing the largest scale of all.

Let us consider the following short-run cost function ${ }^{2}$ :

$$
C(q)=\left\{\begin{array}{ccc}
0 & \text { if } & q \leq 0 \\
a s_{i}+c \frac{k_{i} q}{k_{i}-q} & \text { if } & q>0
\end{array}\right.
$$

with $a, c, k_{i}, s_{i}$ real and positive parameters.

In the first term $s_{i}$ represents the "fitting up" or "setup" costs that, each time a plant is put into operation, are incurred. They may be formed by a common part to all the plants (consider, for instance, electricity costs) and some specific cost to each plant, which is increasing with its dimension (if $k_{i}>k_{j}$, then we should expect that $s_{i}>s_{j}$ ). For convenience we introduce a positive parameter $a$ which is let to vary so that all the fitting up costs are simultaneously proportionally varied. The second term represents variable costs, and becomes infinite as $q \rightarrow k_{i}$ (thus leading to a capacity limit $k_{i}$ ). A constant returns to scale production function will not $\mathrm{do}^{3}$.

For simplicity sake we shall consider only the dynamics between two firms (as the generalization to more firms is standard, but not the related dynamic model) characterized by bounded rationality. In particular, we consider the case in which firms maximize only the profit of the first period that follows their choices. Concerning the production of the concurrent firm they adopt naive expectations. Also, as stated above, we shall assume that both have the same three production options (a so-called symmetric case, although the asymmetric one is quite similar).

This leads us to the study of a two-dimensional model with discontinuities, having both increasing and decreasing jumps. Some results already known for the continuous case, such as the coexistence of several stable cycles, can be extended to the discontinuous one; but not the study of local bifurcations, being

\footnotetext{
${ }^{2}$ As we shall see, the dynamic results of the model are congruent with the choice of a short run cost function.

${ }^{3}$ We should use, for instance, a simplified version of the traditional CES function, as suggested by one of the present authors in several recent publications (see Puu 2005, 2007a,b).
} 
this mainly related to border-collision bifurcations. Completely different are also the results in terms of global analysis of the basins structure. As we shall see, we obtain coexistence of different kinds of stable cycles, and even situations of no single unstable cycle, a specific situation that can only occur with discontinuous dynamical systems.

The paper is organized as follows: In section 2 we introduce the model, considering the simple case of two identical firms (which use identical plants in terms of the capacity limits) and derive the reaction functions of the duopolists. In section 3 we describe the properties of the cycles of the symmetric map $T$ which represents the duopoly (with the same reaction function $\phi$ ). We give the rules to detect all the existing cycles of the duopoly, starting from those of the reaction function $\phi$. Local stability analysis is performed, though the cycles never become unstable, because they "disappear" by border-collision bifurcations when still stable. In section 3.1 we assume an external to the market point of view and analyze the true profits of the duopolists, comparing the different coexisting attractors in terms of profitability for the firms taken separately or together. We show that even if the firms are identical, one of them could be favoured obtaining higher profits. In section 4 we deal with the global properties of the two-dimensional map $T$ in presence of multistability: As no unstable cycle exists, we face the problem of the boundaries of the different basins of the coexistent attractors, which are strictly related to the discontinuities of the reaction functions, and we show how to detect them. Section 5 summarizes our main results and concludes. Proofs are collected in the Appendix at the end of the paper.

\section{The model}

As we restrict our analysis to the duopoly case, let us denote by $x$ and $y$ the outputs produced by the duopolists. Considering one firm, with production $x$. We assume that two plants (producing $x_{1}$ and $x_{2}$ respectively) of limited capacities $k_{1}$ and $k_{2}$, with $k_{2}>k_{1}$, are available. Obviously, in one firm both plants can operate at once, dividing the load of production according to the principle of equal marginal costs. We have

$$
x=x_{1}+x_{2} .
$$

Then, minimizing total production cost $C\left(x_{1}, x_{2}\right)=C\left(x_{1}\right)+C\left(x_{2}\right)$ (with the function given in (1)),

$$
C\left(x_{1}, x_{2}\right)=a s_{1}+c \frac{k_{1} x_{1}}{k_{1}-x_{1}}+a s_{2}+c \frac{k_{2} x_{2}}{k_{2}-x_{2}}
$$

with respect to $x_{1}, x_{2}$ and fixed $x$, we obtain

$$
\left\{\begin{array}{l}
x_{1}=\frac{k_{1}}{k_{1}+k_{2}} x \\
x_{2}=\frac{k_{2}}{k_{1}+k_{2}} x
\end{array},\right.
$$


which, substituted back in (2), yields

$$
C(x)=a\left(s_{1}+s_{2}\right)+c \frac{\left(k_{1}+k_{2}\right) x}{\left(k_{1}+k_{2}\right)-x} .
$$

Denoting this function $C_{3}(x)$, and defining $k_{3}=\left(k_{1}+k_{2}\right), s_{3}=\left(s_{1}+s_{2}\right)$, we automatically have a "third plant" to operate. The options for a firm are thus

$$
C_{i}(x)=a s_{i}+c \frac{k_{i} x}{k_{i}-x} \quad ; \quad i=1,2,3
$$

with $k_{3}=\left(k_{1}+k_{2}\right)>k_{2}>k_{1}$. The choice between the presently three options depends on total costs, as we will see. A higher capacity limit plant implies higher fitting up costs and lower variable costs, so it can be optimal in order to produce high levels of output. Moreover, some levels of output can be produced only using the higher capacity limit technologies. In particular, if the firm wants to produce more then $k_{1}$, it cannot be used the lower capacity limit plant, whereas for a production higher then $k_{2}$ only the higher capacity limit option is available. Graphically the situation is shown in Fig.1where $x^{*}$ denotes

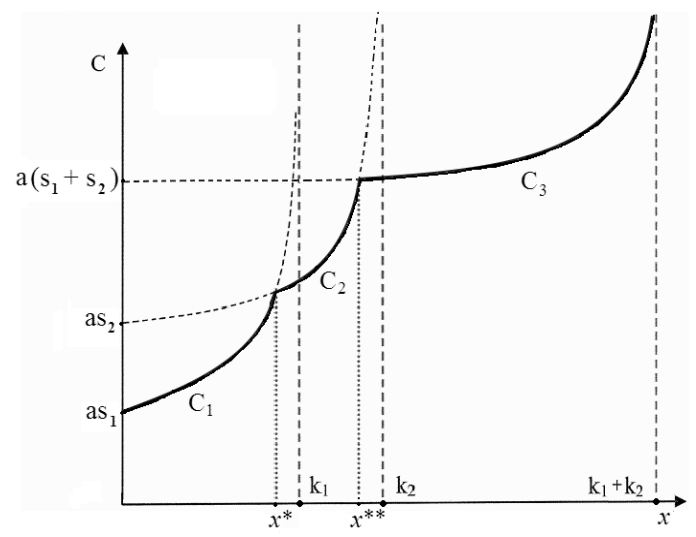

Figure 1: The total cost function corresponding to the lower capacity limit technology is characterized by the lower value of fitting up costs, whereas it increases very quickly with the production because variable costs are very high. The opposite happens using both the plants available: fitting up costs are very high but variable costs are small so it increases very slowly. Using only the higher capacity limit plant we have an intermediate situation.

the output for which the costs for the two plants operated alone break even. Solving the equation $C_{1}\left(x^{*}\right)=C_{2}\left(x^{*}\right)$, i.e., $a s_{1}+c \frac{k_{1} x^{*}}{k_{1}-x^{*}}=a s_{2}+c \frac{k_{2} x^{*}}{k_{2}-x^{*}}$ we get:

$$
x^{*}=\frac{a\left(k_{1}+k_{2}\right)-\sqrt{a^{2}\left(k_{1}+k_{2}\right)^{2}-4 a k_{1} k_{2}\left[a-c \frac{k_{1}-k_{2}}{s_{1}-s_{2}}\right]}}{2\left[a-c \frac{k_{1}-k_{2}}{s_{1}-s_{2}}\right]}
$$


Similarly $x^{* *}$ denotes the output for which the cost for using the larger capacity plant alone breaks even with using both in combination, given by the solution of $C_{2}\left(x^{* *}\right)=C_{3}\left(x^{* *}\right)$, so that we get it from (4) substituting $k_{1}, k_{2}, s_{1}$ and $s_{2}$ with $k_{2}, k_{3}, s_{2}$ and $s_{3}$, respectively. Let us define the following intervals for the quantity of product: $\left.J_{1}=\right] 0, x^{*}\left[, J_{2}=\right] x^{*}, x^{* *}\left[, J_{3}=\right] x^{* *}, k_{3}[$. If the optimal choice $x$ belongs to $J_{i}$ then $C_{i}(x)$ applies. Summarizing, the cost function of the model is given by

$$
C(x)=\left\{C_{i}(x)=a s_{i}+c \frac{k_{i} x}{k_{i}-x} \quad \text { if } \quad x \in J_{i}, \quad i \in\{1,2,3\}\right.
$$

The three different total cost functions give rise to three different marginal cost functions:

$$
M C(x)=\left\{M C_{i}(x)=\frac{d C_{i}(x)}{d x}=c \frac{k_{i}^{2}}{\left(k_{i}-x\right)^{2}} \quad \text { if } \quad x \in J_{i}, \quad i \in\{1,2,3\}\right.
$$

Graphically they are shown in Fig.2. At $x^{*}$ and $x^{* *}$ we have discontinuity points for the marginal cost $M C$ (due to the different slopes at the kink points of the cost function). Assuming an isoelastic demand function

$$
p=\frac{1}{Q_{-1}+x}=\frac{1}{y+x}
$$

where $Q_{-1}$ denotes residual market supply (not under control of the firm itself), that in a duopoly is simple the output of the other firm. Total revenue becomes:

$$
R(x, y)=\frac{x}{y+x},
$$

from which marginal revenue is:

$$
M R(x, y)=\frac{y}{(y+x)^{2}}
$$

It is a decreasing function with respect to $x$, a few examples are shown in Fig.2a,b,c, associated with three different values $y_{a}>y_{b}>y_{c}$, respectively.

We look for the maximum profit (so as to obtain the reaction function of the firm). From the standard computation of the profits, $\Pi(x, y)=R(x, y)-C(x)$, at any fixed value of $y$, say $y^{e}$ the expected level of output of the competitor (as they do not know what will be the production of the other firm they have to make an expectations), the profits of the first firm is given by the envelope function $\Pi^{e}(x)=\max \left\{\Pi_{i}^{e}(x), i=1,2,3\right\}$ of the three profit functions (related with the three plants options) $\Pi_{i}^{e}(x)$ given by

$$
\Pi_{i}^{e}(x)=R\left(x, y^{e}\right)-C_{i}(x) \quad, \quad i=1,2,3
$$

Each of the three profit functions $\Pi_{i}^{e}(x)=R\left(x, y^{e}\right)-C_{i}(x)$ is a unimodal function with only one local maximum point. In fact, considering the first order 
condition, the local extrema are obtained equating marginal revenue to marginal cost, and from the second order conditions it is easy to see that they are all local maxima. Analytically the points are obtained solving

$$
\frac{y}{(y+x)^{2}}=c \frac{k_{i}^{2}}{\left(k_{i}-x\right)^{2}}
$$

and when $0<y<1 / c^{4}$ we have the intersection points $x_{i}^{*} \in J_{i}$ explicitly given by:

$$
x_{i}^{*}\left(y^{e}\right)=k_{i} \frac{\sqrt{\frac{y^{e}}{c}}-y^{e}}{k_{i}+\sqrt{\frac{y^{e}}{c}}} \quad, \quad i=1,2,3
$$

As $k_{1}<k_{2}<k_{3}$ and $x^{*}<x^{* *}$, we also have $x_{1}^{*}\left(y^{e}\right)<x_{2}^{*}\left(y^{e}\right)<x_{3}^{*}\left(y^{e}\right)$. In our case, the envelope function of $x, \Pi^{e}(x)=\max _{i} \Pi_{i}^{e}(x)$ is continuous but not differentiable in all the points, because the points of nonsmoothness $x^{*}$ and $x^{* *}$ of the cost function are also points of nonsmoothness of the maximum profit function. Noticing that a local extrema $x_{i}^{*}\left(y^{e}\right)$ can never occur in a point of nonsmoothness (as this would imply equal constants $k_{i}$ for two plants, which is impossible), we can say that the local extrema of the envelope profit function are necessarily in points in which the function is smooth. Clearly, depending on the value of $y^{e}$, the local maxima may be one or two or three (when they are all involved), and at each time period (or iteration) the choice among such values is the one which gives the maximum expected profit. Thus, in order to get "the best replay" we have to compare the expected profits in the possible cases, given by:

$$
\Pi_{x, i}^{e}\left(y^{e}\right)=\frac{x_{i}^{*}\left(y^{e}\right)}{y^{e}+x_{i}^{*}\left(y^{e}\right)}-a s_{i}-c \frac{k_{i} x_{i}^{*}\left(y^{e}\right)}{k_{i}-x_{i}^{*}\left(y^{e}\right)} \quad i \in\{1,2,3\}
$$

choosing the maximum value, and thus "the plant $i$ ", $i \in\{1,2,3\}$, which gives the maximum. As remarked above, depending on the parameters of the model, and on the expected value $y^{e}$, we may have one, two or even three points of local maxima, which are graphically given by the intersections between the marginal cost function (which is discontinuous, with points of discontinuity in the points of nonsmoothness $x^{*}$ and $x^{* *}$ ) and the marginal revenue, as qualitatively shown in Fig. $2^{5}$. We remark that the number of intersection points of $M R$ and $M C$ may change dynamically as it is a function of the competitor production $y$. For example, starting from the situation in Fig.2(b) with three intersections, when $y$ decreases the $M R$ curve shifts upwards, as in Fig.2(c), whereas if the competitor increases the output produced then the $M R$ curve shifts downwards, as in Fig.2(a). When $y$ is too high, such that $\frac{1}{y}$ approaches $c$, then the profits become negative, but the costraint on the cost function applies, and the production is stopped. Summarizing, given a fixed value of $y$, assuming naive expectations,

\footnotetext{
${ }^{4}$ this constraint is always satisfied in the model as for $y=1 / c$ the solution is $x=0$, which means that the activity is stopped.

${ }^{5}$ It is worth noticing that the same qualitative behavior and final reaction function may be also obtained with any demand function leading to a decreasing Marginal revenue.
} 


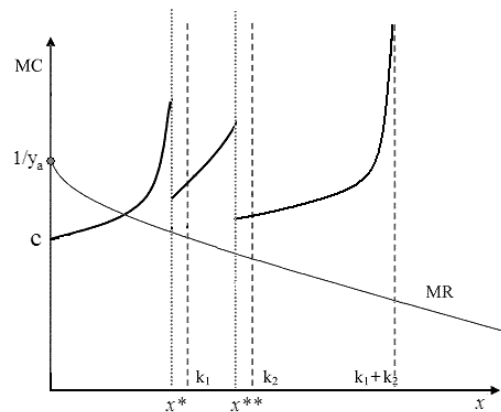

(a)

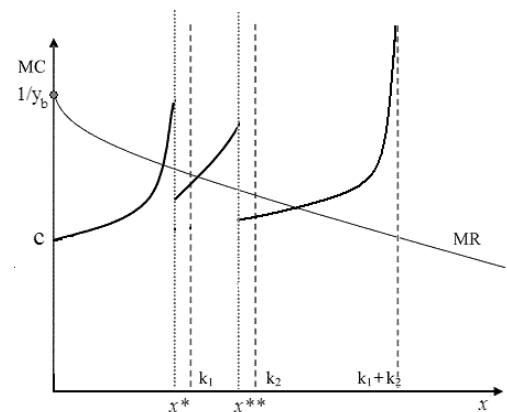

(b)

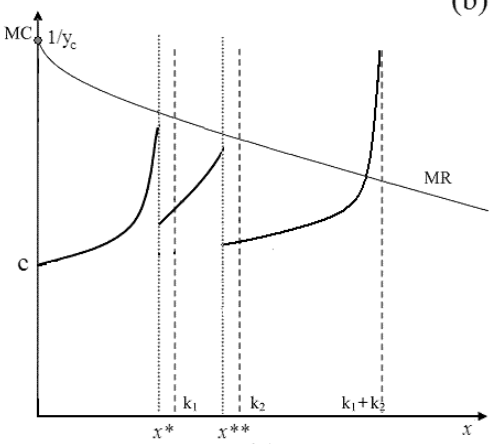

(c)

Figure 2: The Marginal cost curve is formed by three branches (due to the shape of the total cost function in Fig.1). The Marginal revenue function (the thin curve) is strictly decreasing, and starts from the value $1 / \mathrm{y}($ at $\mathrm{x}=0)$. We can have from one (in (a) and (c)) to three (in (b)) intersections.

and denoting the maximum expected profits of the first firm $\Pi_{x}^{e}$, i.e.

$$
\Pi_{x}^{e}=\max \left\{\Pi_{x, i}^{e}(y), i \in\{1,2,3\}\right\}
$$

we have

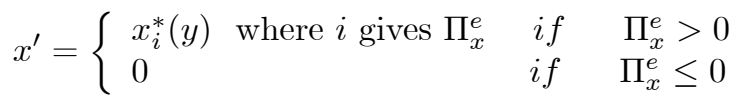

where' denotes the unit-time advancement operator.

The reasoning is similar for the other competitor. There is no reason to assume that their capacity limits $k_{x, i}, k_{y, i}$ are the same. The competitors may not even need to have an equal number of plants to operate. However, to simplify the exposition and the analysis, we shall assume a symmetric case, that is, the two competitors have the same number of plants and the same capacity limits, thus we assume that the parameters $k_{i}$ are the same for the two firms. So we have the best reply $y^{\prime}=y_{i}^{*}\left(x^{e}\right)$ which is obtained as in (10) exchanging the $x$ and $y$ notation, computing the expected profits in the three 
possible cases $\Pi_{y, i}^{e}\left(x^{e}\right)$ as in (11) and selecting the maximum expected profit $\Pi_{y}^{e}=\max \left\{\Pi_{y, j}^{e}(x), j \in\{1,2,3\}\right\}$ for the second duopolist.

\section{Multistability and border-collision bifurcations.}

In the simple duopoly case the reaction functions are given in (12), as well as in the analogue one for the second duopolist. As we have seen, the reaction functions may have one or more discontinuities. A discontinuity happens when, increasing or decreasing the output of the competitor, a different plant operation becomes more profitable than the previous one. In particular, when we are at a discontinuity point what occurs is that we have two different local extrema in the profit envelope function, with the same value of profit, and then there is a change of "best choice" on the right and on the left of the discontinuity point. All the three branches defined above can hence contribute to the final reaction curve. As it is not possible to get the maximum profit in explicit form, it is not possible to find analytically when and where there are discontinuity points in the reaction functions. We have numerical evidence that several cases can occur. However, as we restrict our analysis to the symmetric case, the shape of the reaction functions, when reported in the $(\mathrm{x}, \mathrm{y})$ plane as usual, are symmetric with respect to the main diagonal. The equilibria of the model, or fixed points, are placed (when existing) at the intersections of the two symmetric reaction functions, and are clearly Nash equilibria, or equivalently Cournot equilibria (as in our case the reaction functions depend on only one variable, so that the duopoly model is also a Cournot model). The final reaction curve may be continuous, representing just one particular plant operation (as in Fig.3a) and meaning that the duopolists never use the other alternatives. But we have found that in the generic situation the final reaction curve is formed by several branches (as in Fig.3b,c), representing the use of two or three plants. So, the model is generally described by discontinuous reaction functions. However, it is easy to prove that several properties stated in Bischi et al. 2000 also holds in the discontinuous case, especially those associated with the existence and coexistence of cycles, while this is not true for the bifurcations related with the cycles and the structure of the basin boundaries.

The system formed by (12) and the similar one for the second duopolist, correspond to the particular case of a two-dimensional map having the following structure:

$$
T:\left\{\begin{array}{l}
x^{\prime}=\phi(y) \\
y^{\prime}=\phi(x)
\end{array}\right.
$$

The case of identical firms implies that we can reduce the study of $T$ to the one-dimensional map $\phi(x)$. Indeed, in such a case we have that the properties of the two-dimensional model only depend on the map $F(x)=\phi^{2}(x)$, which, in turn, only depend on those of $\phi(x)$. Due to identical reaction functions, we have that the usual properties of symmetric systems hold, and the relation between the periodic points of $\phi$ and $T$ are easily stated: (proofs in the Appendix)

Proposition 1. In a duopoly game with identical reaction functions then: 


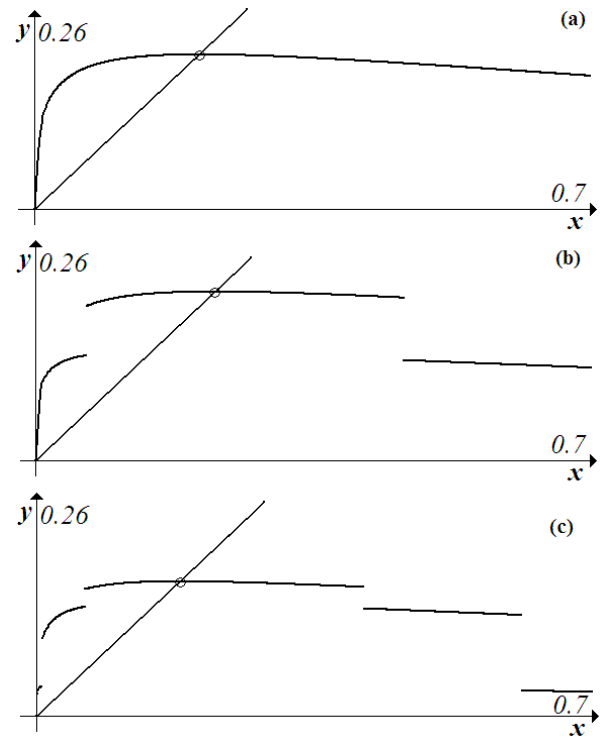

Figure 3: Reactions curves corresponding to different situations. In (a) $k_{1}=0.397$, $k_{2}=0.65, a=0.296, c=0.27, s_{1}=0.37, s_{2}=0.605 . \quad$ In (b) $k_{1}=0.2186$, $k_{2}=0.386, a=0.291, c=0.185, s_{1}=0.3, s_{2}=0.53 . \quad$ In (c) $k_{1}=0.0547$, $k_{2}=0.232, a=0.4376, c=0.185, s_{1}=0.06, s_{2}=0.254479$.

(a) the diagonal $\Delta$ (the straight line $x=y$ ) is a trapping set (i.e. $T(\Delta) \subseteq$ $\Delta)$,

(b) any invariant set I of the phase plane (i.e. such that $T(I)=I$ ), either is symmetric with respect to $\Delta$, or the symmetric one is also invariant.

Proposition 2. Let $\left\{x_{i}\right\}$ be the set of all the periodic points of the map $\phi(x)$, then the points of the Cartesian product $\left\{x_{1}, \ldots, x_{n}\right\} \times\left\{x_{1}, \ldots, x_{n}\right\}$ give all the periodic points of the map $T$ in (13).

These two Properties are very useful because we can obtain the coordinates of the periodic points of $T$ (belonging to the Cartesian product) considering only the dynamics on the diagonal $\Delta$ where the map reduces to $\phi(x)$ :

$$
\phi(x)=\left\{\begin{array}{llll}
k_{i} \frac{\sqrt{\frac{x}{c}}-x}{k_{i}+\sqrt{\frac{x}{c}}} & \text { where } i \text { gives } \Pi_{x}^{e} & \text { if } & \Pi_{x}^{e}>0 \\
0 & \text { if } & \Pi_{x}^{e} \leq 0
\end{array}\right.
$$

where the index $i$ is so chosen that the profit is a maximum, and, as we have seen, $\phi(x)$ may be formed by several disjoint pieces of the three production plant choices.

The following proposition classifies the cycles which we shall call singlygenerated because their existence for the map $T$ is a direct consequence of the existence of one cycle for $\phi(x)$ : 
Proposition 3. (singly-generated cycles) Let $\left\{x_{1}, x_{2}, \ldots, x_{n}\right\}$ be a cycle of $\phi(x)$ of first period $n \geq 1$, then:

If $n$ is odd then T has: (a) one cycle of the period $n$ (on $\Delta$ ); (b) $(n-1) / 2$ cycles of period $2 n$ (external to $\Delta$ ).

If $n$ is even and $n / 2$ is also even then $T$ has $n$ cycles of period $n$ (one on $\Delta$ and $(n-1)$ external to $\Delta)$.

If $n$ is even and $n / 2$ is odd then $T$ has (a) 2 cycles of period $n / 2$ (external to $\Delta)$; (b) $(n-1)$ cycles of period $n$ (one of which on $\Delta$ ).

The following proposition classifies the doubly-generated cycles associated with each pair of cycles of $\phi(x)$ :

Proposition 4. (doubly-generated cycles) Let $\left\{x_{1}, \ldots, x_{n}\right\}$ be a cycle of $\phi(x)$ of first period $n \geq 1$, and $\left\{y_{1}, \ldots, y_{m}\right\}$ a cycle of $\phi(x)$ of first period $m \geq 1$, and let $L$ be the least common multiple between $n$ and $m$, then the cycles of $T$ of type doubly-generated are as follows:

If $n$ and $m$ are odd then $T$ has $\frac{n \cdot m}{L}$ cycles of period $2 L$.

If $n$ or/and $m$ are even then $T$ has $\frac{2 n \cdot m}{L}$ cycles of period $L$.

Particular attention must be paid to the fixed points of $T$. Due to the complex expression of the reaction functions it is not possible to obtain the explicit analytic formulation of the equilibria. Clearly $\left(x^{*}, y^{*}\right)$ is a fixed point of $T$ iff $T\left(x^{*}, y^{*}\right)=\left(\phi\left(y^{*}\right), \phi\left(x^{*}\right)\right)=\left(x^{*}, y^{*}\right)$. It follows that if $x_{1}$ is a fixed point of $\phi(x)$, then $\left(x_{1}, x_{1}\right)$ is a fixed point of $T$ (of singly-generated type) belonging to the diagonal $\Delta$. When we have two fixed points of $\phi$, say $x_{1}$ and $x_{2}$, then $T$ has two fixed points of singly-generated type, $B=\left(x_{1}, x_{1}\right)$ and $A=\left(x_{2}, x_{2}\right)$, belonging to the diagonal $\Delta$. Further, by Proposition 4 a $2-$ cycle of $T$ of doubly-generated type external to $\Delta$ is obtained, with periodic points $\left\{\left(x_{1}, x_{2}\right),\left(x_{2}, x_{1}\right)\right\}$.

An important feature of this model is that the bifurcations of fixed points do not occur via the modulus of the eigenvalues (which is the standard way in smooth maps). Instead, here a fixed point bifurcates via border-collision: It disappears when merging with a point of discontinuity. Due to the flat shape of the reaction functions $\phi$ in the fixed points and in the cycles (as shown also in Fig.3), the dynamics are very fast ${ }^{6}$, and all the cycles bifurcate via bordercollision. What occurs "after" depends on the slopes of the two branches of the reaction function involved in the upper and lowed branches at the discontinuity point. In our model we have always detected stable cycles of low period (three or four) An example is shown in Fig.4a, at which $\phi(x)$ has only one attractor, the asymptotically stable fixed points $P^{*}$ (using the second technology, with capacity limit $k_{2}$ ) and increasing the parameter $c$ the fixed point merges with the discontinuity point $\xi_{2}$ (at $c \simeq 0.1789$ ). Soon after the collision, at $c=0.182$, a stable 4 -cycle appears on the diagonal $\Delta$, shown in Fig.4b, with periodic points (in the dynamic order) $A, B, C$ and $D$, existing for a very narrow interval of values of $c$. The periodic point $D$ corresponds to a quantity produced using the first technology. Then a second border collision bifurcation (a periodic

\footnotetext{
${ }^{6}$ this is congruent with the short run horizon, each competitor reacts in just a few periods.
} 


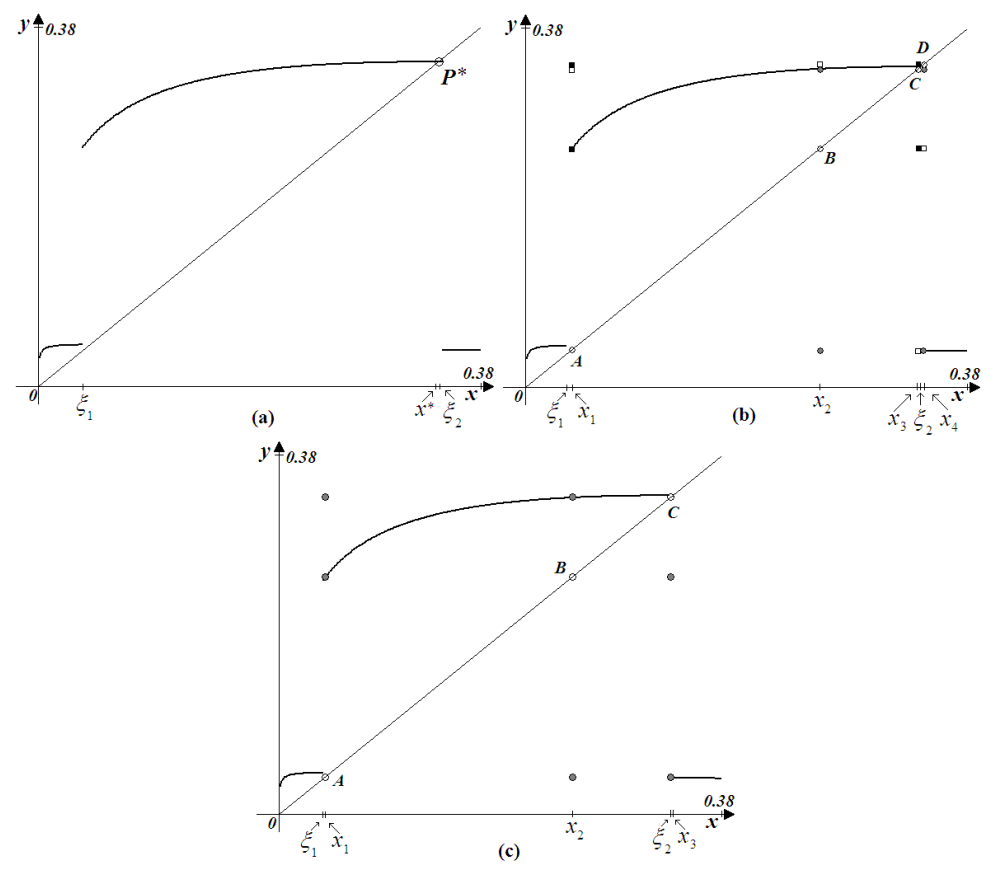

Figure 4: Attractors at $k_{1}=0.0547, \quad k_{2}=0.676, \quad a=0.5835, \quad s_{1}=0.045$, $s_{2}=0.556$. In (a) $c=0.175$, a fixed point $P^{*}$. In (b) $c=0.182,4$ coexisting 4-cycles. The cycle $\{A, B, C, D\}$ on the diagonal is given by $\left(x_{i}, x_{i}\right), i=1, \ldots, 4$ respectively where $x_{1} \simeq 0.0395153713, x_{2} \simeq 0.25244, x_{3} \simeq 0.33742446$ and $x_{4} \simeq 0.339785$. In (c) $c=0.185$ a 3 -cycle on $\Delta$ coexists with a 6 -cycle outside the diagonal. The 3 -cycle is given by points $\left(x_{i}, x_{i}\right), i=1,2,3$ corresponding to $A \simeq(0.039468,0.039468)$, $B \simeq(0.2509525,0.2509525)$, and $C \simeq(0.33557315,0.33557315)$ respectively. Points belonging to the same cycle are marked with the same symbol. The $\xi_{i}$ denote the discontinuity points.

point merges with the discontinuity point $\xi_{2}$ ) causes the disappearance of the 4 -cycle leading to a stable 3 -cycle on the diagonal for $T$, which is shown in Fig.4c, with periodic points (in the dynamic order) $A, B$ (using the lower capacity limit technology) and $C$ (using the second technology). As $c$ is further increased, the periodic point $A$ approaches the first discontinuity point and at $c \simeq 0.211$ the merging occurs $\left(x_{1}=\xi_{1}\right)$, leading to the disappearance of the 3 -cycle and appearance of another 4 -cycle, now with two periodic points associated with the first technology ( $A$ and $D$, see Fig.5a) while the periodic points $B$ and $C$ are associated with the second technology. Finally, the last border-collision bifurcation occurring as $c$ increases causes the disappearance of the 4-cycle and appearance of another stable fixed point, $Q^{*}$, now associated with the first technology (see Fig.5b). Thus increasing the variable costs the 
dynamic behavior goes towards a gradual substitution of the second technology by the lower technology. This happens because the variable costs become more and more relevant and the higher revenue related with an higher capacity limit technology does not balance the higher costs due to an higher level of production.

Regarding the complete attracting sets existing in the market, when the function $\phi(x)$ has a three cycle on $\Delta$ then the Cartesian product $\left\{x_{1}, x_{2}, x_{3}\right\} \times$ $\left\{x_{1}, x_{2}, x_{3}\right\}$ includes 9 points, and besides the 3 -cycle we have, from Proposition 3 , a stable 6 -cycle external to $\Delta$. In this example the border-collision may recall the border-collision bifurcation described in Hommes 1991 in the continuous case, but here there is no relation (no continuity) between the periodic points before and after the bifurcation.

Considering the cases in which the map $\phi(x)$ has an attracting 4-cycle, from Proposition 3 we have that 4 coexisting cycles, all of period 4 , must exist, one on the diagonal $\Delta$ and three outside (see Fig.4c and Fig.5a). We have not yet
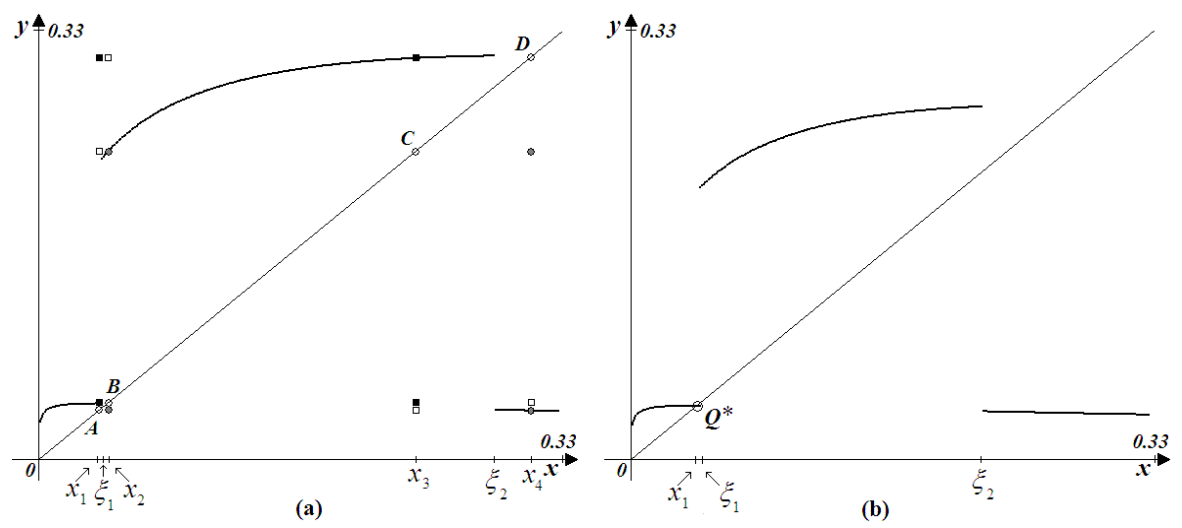

Figure 5: Attractors at fixed parameters values as in Fig.4 and In (a) $c=0.23,4$ coexisting 4-cycles. The cycle $\{A, B, C, D\}$ on the diagonal is given by $\left(x_{i}, x_{i}\right)$, $i=1, \ldots, 4$ respectively where $x_{1} \simeq 0.038267674, x_{2} \simeq 0.043707, x_{3} \simeq 0.23845$ and $x_{4} \simeq 0.311127664$. Points belonging to the same cycle are marked with the same symbol. The symmetric cycle is marked by white squares, the cycle $\mathcal{C}_{3}$ is marked by black circles and its symmetric one, $\mathcal{C}_{4}$, by black squares. The $\xi_{i}$ denote the discontinuity points.

rigorously considered the problem of the stability/instability of the cycles of $T$. In the case in which $\phi(x)$ is piecewise smooth, with one or several discontinuity points, we consider that cycles of $\phi(x)$ have periodic points in which the function is differentiable, that is, we do not consider the bifurcations related to the appearance/disappearance of such cycles (which in our case occur by bordercollision bifurcations), here we are only interested in their local stability, once they exist. As we have shown above, any cycle of $\mathrm{T}$ is related to one (if singlygenerated) or two (if doubly-generated) cycles of $\phi(x)$, and it is easy to see, considering the Jacobian matrix of $T$, that the following proposition holds (and 
this proves the stability results in the examples given above).

Proposition 5. Let $X=\left\{x_{1}, \ldots, x_{n}\right\}$ be a cycle of $\phi(x)$ of first period $n \geq 1$, and $Y=\left\{y_{1}, \ldots, y_{m}\right\}$ a cycle of $r(x)$ of first period $m \geq 1$, then:

If $X$ is asymptotically stable (resp. unstable) for $\phi(x)$ with eigenvalue $\lambda$, $|\lambda|<1$ (resp. $|\lambda|>1$ ) then all the singly-generated cycles associated with $X$ are asymptotically stable (unstable), star nodes for $T$ with eigenvalues $\zeta_{1}=\zeta_{2}=\lambda$.

If $X$ and $Y$ are both asymptotically stable for $\phi(x)$, with eigenvalues $\lambda$ and $\mu(|\lambda|<1,|\mu|<1)$, then all the doubly-generated cycles associated with $X$ and $Y$ are asymptotically stable nodes for $T$ with eigenvalues $\zeta_{1}=\lambda, \zeta_{2}=\mu$.

If $X$ or $Y$ is unstable for $\phi(x)$, with eigenvalues $\lambda$ and $\mu,|\lambda|<1$ and $|\mu|>$ 1 , then all the doubly-generated cycles associated with $X$ and $Y$ are unstable for $T$, of saddle type, with eigenvalues $\zeta_{1}=\lambda, \zeta_{2}=\mu$.

If $X$ and $Y$ are both unstable for $\phi(x)$, with eigenvalues $\lambda$ and $\mu(|\lambda|>1$, $|\mu|>1)$, then all the doubly-generated cycles associated with $X$ and $Y$ are unstable nodes for $T$, with eigenvalues $\zeta_{1}=\lambda, \zeta_{2}=\mu$.

\subsection{Comparison among coexisting attractors.}

As we have seen in the previous section, at each period the competitors choose the maximum expected profit, and we have found short period dynamics. It is now interesting to analyze what happens to a firm globally, say after a few periods, computing the real profit, associated with the realized choices (a kind of a posteriori analysis). During the iteration process the first firm "assumes" that the other one keeps to the quantity of the previous period (say $y_{t}$ ) and decides its own production (say $x_{t+1}$ ) in a process which selects maximum profit. The same is true for the second firm. But we did not dynamically evaluate the actual, or real, profits of the firms. So in this section we compute the profits obtained by each firm during one period (i.e. in some stable state) and the market formed by the two competitors. Let us define $P_{x}(A)$ the real profit of the first firm in a point $A=(x, y)$, i.e. with such quantities. We have

$$
P_{x}(A)=\frac{x}{x+y}-C(x) \quad, \quad P_{y}(A)=\frac{y}{x+y}-C(y)
$$

where $C(x)$ is the cost function defined in (5), $C(x)=a s_{i}-c \frac{k_{i} x}{k_{i}-x}$ for a suitable $i \in\{1,2,3\}$, and similarly for the second firm. The parameters in the cost function depend on the values of the productions and thus on the branches of the reaction functions involved, which may also be different. We also define $P_{x}^{(m)}(A)$ as the sum of the profits of the first firm in the $m$ periods of the trajectory starting from $A$. The same for $P_{y}^{(m)}(A)$. Finally, we are interested in the industry profit, $\operatorname{IP}(A)$ in a point $A=(x, y)$, which is the sum of both the firms in $A$, that is, for some suitable $i, j \in\{1,2,3\}$ :

$$
\begin{aligned}
I P(A) & =P_{x}(A)+P_{y}(A)=1-C(x)-C(y)= \\
& =1-a\left(s_{i}+s_{j}\right)-c\left(\frac{k_{i} x}{k_{i}-x}+\frac{k_{j} y}{k_{j}-y}\right)
\end{aligned}
$$


and the profit $I P^{(m)}(A)$ is the sum of the industry profits in the $m$ periods of the trajectory starting from $A$.

Clearly in a point on $\Delta$ we have $P_{x}(A)=P_{y}(A)$ because $x=y$ (and thus $k_{i}=k_{j}$ and $\left.s_{i}=s_{j}\right)$, so that we also have $P_{x}^{(n)}(A)=P_{y}^{(n)}(A)=\frac{1}{2} I P^{(n)}(A)$ for each $n>0$.

If we consider a point $E=(x, y)$ which does not belong to the diagonal, the profits $P_{x}(E)$ and $P_{y}(E)$ are different, and if we take the symmetric point $E^{\prime}=(y, x)$ we clearly have $P_{x}\left(E^{\prime}\right)=P_{y}(E)$ and $P_{y}\left(E^{\prime}\right)=P_{x}(E)$, because in $E$ the first (resp. second) firm produces the same quantity (and thus uses the same plant capacities) as the second (resp. first) firm in $E^{\prime}$. It follows that the industry profit in symmetric points is the same:

$$
\begin{aligned}
I P(E) & =P_{x}(E)+P_{y}(E)=1-C(x)-C(y)= \\
& =P_{y}\left(E^{\prime}\right)+P_{x}\left(E^{\prime}\right)=I P\left(E^{\prime}\right)
\end{aligned}
$$

Moreover, in symmetric points both firms have the same sum of profits, which also is the same value as the industry profit:

$$
P_{x}(E)+P_{x}\left(E^{\prime}\right)=P_{y}\left(E^{\prime}\right)+P_{y}(E)=1-C(x)-C(y)
$$

Let us consider what occurs in the analysis ex post, after one period, in the case shown in Fig.4c. We shall see that the profit of both firms after 6 periods is the same in both the different attractors. In fact, let $A=\left(x_{1}, x_{1}\right)$ be a point of the 3 -cycle on $\Delta$. Then we know that $P_{x}^{(n)}(A)=P_{y}^{(n)}(A)=\frac{1}{2} I P^{(n)}(A)$ for each $n>0$. Further let $E=\left(x_{1}, x_{2}\right)$ be a point of the 6-cycle outside $\Delta$. The 6 -cycle is formed by 3 pairs of symmetric points, in each pair of which the sum of the profits is the same. After 6 periods every point is visited once, so that the total profit after 6 periods is equidistributed: $P_{x}^{(6)}(E)=P_{y}^{(6)}(E)=\frac{1}{2} I P^{(6)}(E)$. But it can be proved that these values are equal, i.e. $I P^{(6)}(A)=I P^{(6)}(E)$ and $I P^{(3)}(A)=\frac{1}{2} I P^{(6)}(E)$, so that $P_{x}^{(6)}(E)=P_{y}^{(6)}(E)=P_{x}^{(3)}(A)+P_{y}^{(3)}(A)$. This comes from the following:

Proposition 6. Let $X=\left\{x_{1}, \ldots, x_{n}\right\}$ be a cycle of $\phi(x)$ of first period $n>1$, and $p=n(n-1)$, then

(a) the sum of the industry profits of the $p$ periodic points of $T$ singly generated from $X$ (and external to $\Delta$ ) is equal to $I P^{(p)}\left(x_{1}, x_{1}\right)$, i.e. $(n-1)$ times the industry profit $I P^{(n)}$ on the n-cycle $X$ on the diagonal;

(b) the sum of the profits of each firm (first and/or second) of the p periodic points of $T$ singly generated from $X$ (and external to $\Delta$ ) is equal to $P_{x}^{(p)}\left(x_{1}, x_{1}\right)=P_{y}^{(p)}\left(x_{1}, x_{1}\right)=\frac{1}{2} I P^{(p)}\left(x_{1}, x_{1}\right)$.

Thus in the case shown in Fig.4c, with a 3 -cycle on $\Delta$ and a 6 -cycle outside, after 6 periods we get the same industry profit, both starting from a periodic point on $\Delta$ and starting from a periodic point outside the diagonal. Indeed the case $n=3$ is particular, because it is the unique period for which we have only one singly generated cycle outside the diagonal. In general, outside $\Delta$ there are more coexisting singly-generated cycles, and the sum of the profits on different cycles may differ (although globally Proposition 6 above holds). 
Moreover, we may have coexisting cycles on the diagonal. In such cases, when $m$ cycles (with $m>1$ ) belong to $\Delta$, outside the diagonal we have not only singly-generated cycles, but also doubly-generated cycles, and we can find interesting economic situations. Note that when comparing profits corresponding to different cycles, we consider a number of periods equal to the least common multiple among the periodicity of the attractors. This is just to be sure that all the points of each cycle are visited the same number of times, otherwise the comparison is not informative because some points would be more important than others.

Now let us consider the case shown in Fig.5a (or equivalently the case shown in Fig.4b) where we have a 4-cycle on $\Delta$ and three stable coexisting 4-cycles outside the diagonal. Also in this case we have that the industry profit IP after 4 periods is the same in all the coexisting cycles. Indeed this property holds whenever we apply Proposition 3 to a cycle of period $n$ with $n$ and $\frac{n}{2}$ even (so that the model has $n$ coexisting $n$-cycles). To see this let us denote by $\left(x_{i}, x_{i}\right), i=1, \ldots, n$ the periodic points of the $n$-cycle on the diagonal, such that $x_{i+1}=\phi\left(x_{i}\right)$. Then all the $n$ coexisting cycles, say $\mathcal{C}_{k}$ for $k=1, \ldots, n$, have periodic points given by

$$
T^{i}\left(x_{j}, x_{k}\right), \quad i=1, \ldots, n
$$

for some suitable $j$ and $k$ (for example the cycle on $\Delta$ is given by $j=k=1$ ). But considering that $T\left(x_{j}, x_{k}\right)=\left(\phi\left(x_{k}\right), \phi\left(x_{j}\right)\right)=\left(x_{k+1}, x_{j+1}\right)$ the quantities involved in $n$ steps from the two competitors are given by

$$
\begin{aligned}
x_{j+1}, \ldots, x_{j+n-1}, x_{j+n}( & \left.=x_{j}\right) \\
x_{k+1}, \ldots, x_{k+n-1}, x_{k+n}( & \left.=x_{k}\right)
\end{aligned}
$$

thus whichever are $j$ and $k$ the industry profit $I P^{(n)}$ takes the same value in any cycle $\mathcal{C}_{k}$ (for $k=1, \ldots, n$ ) as it is given by

$$
\begin{aligned}
I P^{(n)}\left(\mathcal{C}_{k}\right) & =I P^{(n)}\left(x_{j}, x_{k}\right)=\sum_{i=1}^{n}\left[1-C\left(x_{j+i}\right)-C\left(x_{k+i}\right)\right] \\
& =n-\sum_{i=1}^{n}\left[C\left(x_{j+i}\right)+C\left(x_{k+i}\right)\right] \\
& =n-2 \sum_{i=1}^{n} C\left(x_{i}\right)
\end{aligned}
$$

where $C(x)$ is the cost function defined in (5).

Regarding the profit of the two duopolists after $n$ periods, from the results stated above for symmetric points we know that cycles having symmetric points have the industry profit equidistributed between the two duopolists: $P_{x}^{(n)}=$ $P_{y}^{(n)}$. Moreover, in this case this is also equal to the profit after $n$ periods of the cycle on $\Delta$ (i.e. $\left.P_{x}^{(n)}=P_{y}^{(n)}=P_{x}^{(n)}\left(x_{1}, x_{1}\right)=P_{y}^{(n)}\left(x_{1}, x_{1}\right)\right)$. While for the cycles with asymmetric periodic points the profit after $n$ periods may not be 
equidistributed between the two duopolists, and this is an example in which a duopolist is "dominant" in the market, although the industry profit is the same.

In the case of Fig.4c, at the end of the sixth period the industry profit is equidistributed whatever is the attractor. In the case of Fig.5a (or equivalently of Fig.4b) the industry profit is still equidistributed on the cycle on the diagonal and on the symmetric cycle, while this is not the case for the other two 4-cycles. The cycle $\mathcal{C}_{3}$ has three periodic points below the diagonal, where $x>y$, and after four iterations it results that the firm whose production is denoted by " $x$ " obtains an higher profit than the other $\left(P_{x}^{(4)}\left(\mathcal{C}_{3}\right)=1.452242>P_{y}^{(4)}\left(\mathcal{C}_{3}\right)=\right.$ 0.925792). The opposite is true for the cycle $\mathcal{C}_{4}$ (whose points are symmetric with respect to those of $\mathcal{C}_{3}$ ).

The analysis performed in this section has no consequences for the choices of the duopolists because, in taking their decisions they only consider the today's expected profit. We have shown that starting from identical firms (both in terms of technologies and rationality, as both have naive expectations on the competitor production), multistability causes the coexistence of attractors in which one firm obtains more profit than the other one.

\section{Global Analysis}

In all our examples, except for the wide range of existence of one single fixed point, we have seen the phenomenon of multistability. Indeed the shape of $\phi(x)$ far from the origin is "quite flat" and the periodic points of the one-dimensional function $\phi(x)$ are all in branches of the function such that $\left|\phi^{\prime}(x)\right|<1$. From Proposition 5 it follows that all the coexisting cycles of $T$ are asymptotically stable. In particular, $T$ cannot have saddle cycles (nor repelling nodes, and we recall that complex eigenvalues cannot occur in the class of maps having separate second iterate). This is the main difference with respect to the class of maps in the continuous case: All the existing cycles are asymptotically stable and their basins fill in the phase-plane. A natural question then arises: having no unstable cycles (the stable sets of which usually give the boundaries of different basins), what are now the boundaries? The answer to this question, related to the global dynamic behavior, comes from the discontinuity points.

In order to detect the basins of attraction in our model, the behavior of the points $(x, y)$ of the phase plane of $T$ can be better analyzed by using the second iterate (i.e. the map $T^{2}$ ). As all the existing cycles are asymptotically stable, it follows that the critical points of the function $\phi(x)$, or $\phi^{2}(x)$ do not have dynamic relevance because such critical points converge to some stable cycle. Of primary importance are the discontinuity points of the function $\phi^{2}(x)$, which depend on those of $\phi(x)$. In fact, the discontinuity points of $\phi^{2}(x)$ consist in all the discontinuity points of $\phi(x)$ and in all their rank-1 preimages (when existing). We recall that a discontinuity point, say $x=\xi$, of the reaction function $\phi(x)$ corresponds to the discontinuity line of equation $x=\xi$ in the phase plane for the two-dimensional map $T$ and, due to the symmetry, also $y=\xi$ is a discontinuity line. 
In general, at each discontinuity of the reaction function $\phi(x)$, say at $x=\xi$, we have to consider the two values associated with the "jump" of the function, say $\phi_{l}(\xi)$ (lower value) and $\phi_{u}(\xi)$ (upper value), and in the study of discontinuous maps the discontinuity points and their lower/upper values take the role of the "critical points" in the Julia sense, as considered in Mira et al., 1996. If the asymptotic behavior of the two values $\phi_{l}(\xi)$ and $\phi_{u}(\xi)$ is the same, then the vertical line $x=\xi$ (discontinuity for $T$ ) plays no role in the basin boundaries. Vice-versa, if the asymptotic behavior of the two values $\phi_{l}(\xi)$ and $\phi_{u}(\xi)$ are different, then the vertical line $x=\xi$ is a boundary of basins. This is clearly due to the fact that all the points in a right/left neighborhood of the discontinuity at $x=\xi$ have the same asymptotic behavior of the two values $\phi_{u}(\xi)$ and $\phi_{l}(\xi)$, or $\phi_{l}(\xi)$ and $\phi_{u}(\xi)$ (depending on the graph of the function). Such behaviors are to be considered in the function $\phi^{2}(x)$, for which $x=\xi$ is still a discontinuity point, and thus $\phi_{l}^{2}(\xi)$ and $\phi_{u}^{2}(\xi)$ the values at the jump.

Moreover if $x=\xi$ is a discontinuity point of $\phi(x)$ which is a separator of different basins, then also any one of its rank-1 preimages is a separator of basins, because points in a suitable right/left neighborhood of a preimage of $x=\xi$, say at $x=\xi_{1}^{-1}$, are mapped in one iteration in a right/left (or left/right) neighborhood of $x=\xi$, and thus have a different asymptotic behavior. The same reasoning can clearly be applied to the discontinuity points of the function $\phi^{2}(x)$. If one of the discontinuity points of $\phi^{2}(x)$ is a boundary of basins, then the same property holds for all the existing preimages, of any rank.

The asymptotic behavior of $x$, determined by the function $\phi^{2}(x)$ i.e. the segments of basins for this one-dimensional function, may be associated with the $x$-axis in our two-dimensional phase plane. In this symmetric case, we also have that the asymptotic behavior of $y$ is determined by the same function, so that the same segments of basins may be associated to the $y$-axis. Then we form the Cartesian product, so that we get the basins of the map $T^{2}$, from which we easily obtain those of $T$.

Regarding the points of the boundary itself, we confine the discussion to the discontinuity points. In each discontinuity point $x=\xi$ we have considered the two values $\phi_{l}(\xi)$ and $\phi_{u}(\xi)$ without saying which one is assumed in the discontinuity point $\xi$. Thus a point in a vertical boundary $x=\xi$ may behave as a point in its right/left neighborhood, depending on which value is taken at $x=\xi$. Similarly, in a horizontal boundary say $y=\xi$, we have that any point of a boundary may belong to any one of the basins of which it is a boundary, depending on the values associated at the discontinuity.

This generic description may be better understood by looking at the basins of some of the examples already seen in the previous sections. Let us return to the case represented in Fig.5a, where we have four coexisting attractors of $T$ (a 4-cycle on the diagonal, and three 4-cycles outside). In Fig.6a we show the graph of the function $\phi^{2}(x)$ with the discontinuity points at $x=\xi_{1}$ and $x=\xi_{2}$ as in the graph of $\phi(x)$ (see Fig.5a). Two more discontinuity points of $\phi^{2}(x)$ exist in $x=\xi_{2}^{-1}$ and $x=\xi_{1}^{-1}$, the rank-1 preimages of the discontinuity points $x=\xi_{2}$ and $x=\xi_{1}$, respectively. From the graph of $\phi^{2}(x)$ in Fig.6a we can see that the two values $\phi_{l}^{2}\left(\xi_{1}\right)$ and $\phi_{u}^{2}\left(\xi_{1}\right)$ (extrema at the jump in $\xi_{1}$ ) have 

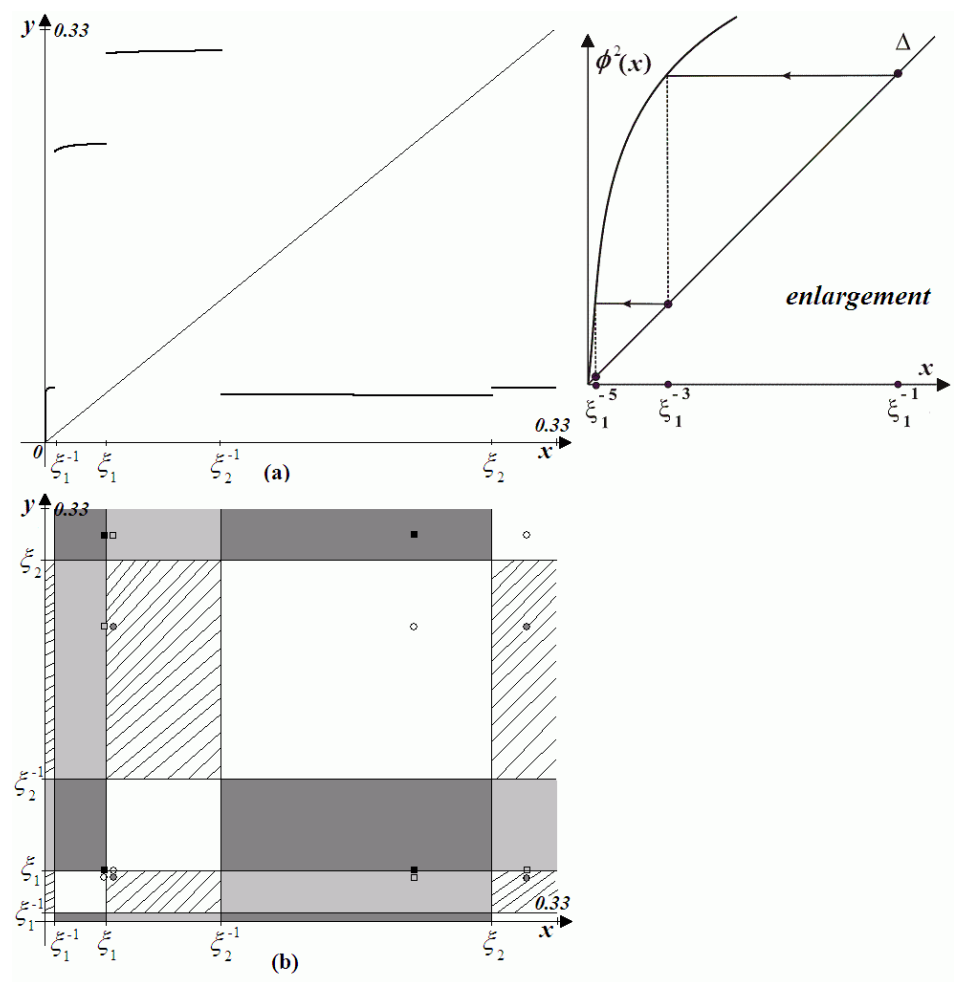

Figure 6: In (a) we show $\phi^{2}(x)$ and in (b) the basins of attraction corresponding to the situation shown in Fig. $5 \mathrm{a}$. The basin of the cycle on $\Delta$ is in white. The lined and gray tonalities give the basins of the coexisting three 4 -cycles outside $\Delta$.

different asymptotic behavior: The lower value tends to the 2-cycle of points $(A, C)$ in Fig.5a, while the upper value converges to the other 2-cycle (points $B, D$ in Fig.5a). Thus the line $x=\xi_{1}$ is a boundary of basins and the same occurs at the discontinuity in its rank-1 preimage, at $x=\xi_{1}^{-1}$ (which is quite close to zero). Similarly the extrema at the jump in $\xi_{2}$ have different asymptotic behaviors, thus the lines $x=\xi_{2}$ and $x=\xi_{2}^{-1}$ are boundaries of basins, and no further preimges of $\xi_{2}^{-1}$ exist. In the enlargement of Fig. 6 we show qualitatively that a sequence of preimages at $x=\xi_{1}^{-(1+2 k)}$, for $k=1,2, \ldots$ must exist, rapidly approaching the origin. Thus boundaries of basins (though not discontinuity points) are all the preimages $x=\xi_{1}^{-(1+2 k)}$ for any $k>1$ (even if these points are not visible in Fig.6 because they are too close to zero).

To summarize, on the $x$-axis we have intervals separated by $\xi_{1}, \xi_{1}^{-1}, \xi_{1}^{-(1+2 k)}$, the points of which tend alternatively (for $\phi^{2}(x)$ ) to different $2-$ cycles on $\Delta$. The same holds about the $y$-axis, with the same intervals and the same asymptotic behaviors, so that the horizontal lines $y=\xi_{2}, y=\xi_{2}^{-1}, y=\xi_{1}, y=\xi_{1}^{-1}$, 
$y=\xi_{1}^{-(1+2 k)}$ approaching zero, belong to boundaries of basins. The Cartesian product is made up by rectangles, in which we can easily see the four different basins for the map $T$, as shown in Fig.6b (though the rectangles close to the coordinate axes are not visible).

In Fig.7 we show the basins of attraction of the case represented in Fig.4c, where we have only two coexisting attractors of $T$ (a 3 -cycle on the diagonal, and a symmetric 6 -cycle outside). From Fig.4c we can see that the graph of the function $\phi^{2}(x)$ must have the discontinuity points at $x=\xi_{1}$ and $x=\xi_{2}$ as in the graph of $\phi(x)$ and three more discontinuity points: one at $x=\xi_{2}^{-1}$, rank-1 preimage of the discontinuity points $x=\xi_{2}$, and two other points are the rank-1 preimages of $x=\xi_{1}$, respectively in $x=\xi_{1}^{\prime-1}$ and $x=\xi_{1}^{-1}$. This last one is very close to zero and we know that a sequence of preimages at $x=\xi_{1}^{-(1+2 k)}$, for $k=1,2, \ldots$ must exist, rapidly approaching the origin. As the attractor of $\phi^{2}(x)$ is always the same 3 -cycle, we have that any point $x$ converges to the 3 -cycle and similarly for the symmetric case: any point $y$ converges to the 3 -cycle. Thus all the lines issuing from the discontinuity points (on the $x$-axis and $y$-axis) give a Cartesian product which separates rectangles whose points may converge to the two attractors. That is, any point $(x, y)$ of the phase plane may have coordinates which converge in phase (to the $3-$ cycle, white points in Fig.7) or out of phase (to the 6-cycle, gray points in Fig.7). And boundaries of basins (though not discontinuity points) are all the lines issuing from the preimages $x=\xi_{1}^{-(1+2 k)}$ and $y=\xi_{1}^{-(1+2 k)}$ for any $k>1$ (even if these points are not visible in Fig.7 because too close to zero).

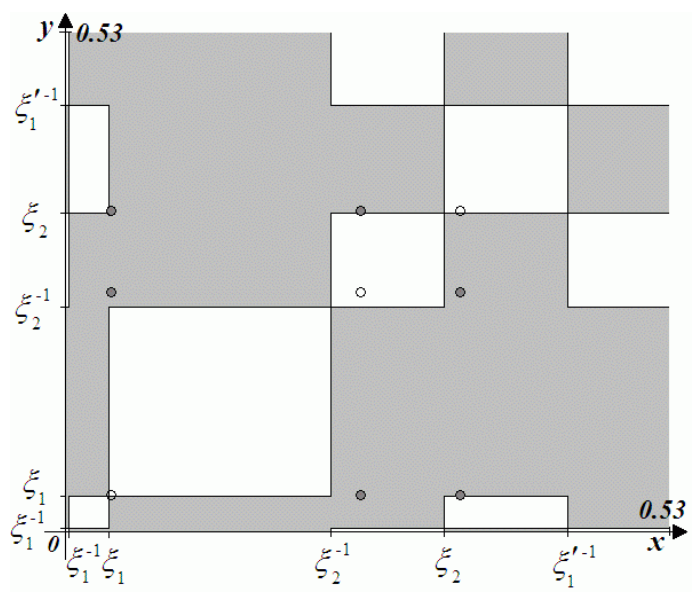

Figure 7: The basins of attraction corresponding to the situation shown in Fig. 4c. In white the basin of the 3 -cycle on $\Delta$, in grey the basin of the 6 -cycle. 


\section{Conclusions}

We can distinguish between two kinds of conclusions: economically or mathematically relevant. From an economic point of view, in this study we have considered a Cournot duopoly under an isoelastic demand function and cost functions with built-in capacity limits. The special feature is that each firm is assumed to operate multiple plants. Each firm has two plants with different capacity limits, which may be used one by one or in combination. It has three cost options, the third being to run both plants, dividing the production load according to the principle of equal marginal costs. As a consequence the marginal cost functions come in three disjoint pieces, so the reaction functions, derived on basis of global profit maximization, can consist of several disjoint pieces. We are thus faced with discontinuous, piecewise smooth, reaction functions.

We have analyzed the case of identical firms, characterized by a symmetric two-dimensional map. One peculiar feature of the duopoly model is that the changes in the coexistent attractors never occur due to some stable cycle becoming unstable. Instead, all the bifurcations are due to border collision; merging of some cycle with the discontinuity points. We have seen that several stable cycles may coexist, and how to detect all them starting from the reaction function. Another property is that from any point of the phase space a trajectory converges to some stable situation, given the total absence of unstable cycles. This perhaps reflects an important realistic situation. That is, in the market (or real situation) in which the two competitors are involved, there is no unstable equilibrium (which may also be difficult to justify), but only a few different stable configurations (either in equilibrium points or in stable cyclical occurrence).

We have seen how to compare the coexistent periodic attractors in terms of real profits, looking "from outside" the behavior of the firms in a few periods. We have performed a global analysis of the phase plane, showing that the boundaries of the different basins are related with the discontinuities of the reaction functions, explaining how to detect the boundaries.

We limited our analysis to a model with two identical competitors. However, it is worth noting that a similar study may be performed with two non-identical firms or more firms, increasing the analytical complexity of the model but not the qualitative dynamic results.

From a mathematical point of view, our work belongs to a recent stream of literature focused on the study of maps with discontinuities. This field still present open problems, for instance: once that a point cycle merges into a border, how can we predict or know what will occur? The first studies in this direction, for one-dimensional maps, are due to Leonov $(1959,1962)$, Mira (1978, 1987), the recent papers by Avrutin and Shanz (2006) and Avrutin et al. (2006) are mainly related with positive (increasing) jumps in the discontinuity points. The effects of those related to both increasing and decreasing jumps (which is of interest for our model) is still under study. 


\section{Appendix.}

\section{Proof of Proposition 1.}

The proof of (a) is immediate: let $(x, x) \in \Delta$ then $T(x, x)=(\phi(x), \phi(x)) \in$ $\Delta$. To prove (b) let us denote by $S$ the symmetric operator such that $S(a, b)=$ $(b, a)$, then, from the definition of $T$, we have $S(T(x, y))=T(S(x, y))$. Now let $I$ be an invariant set of $T$, so that $T(I)=I$, then $S(T(I))=T(S(I))=S(I)$ holds. It follows that either $S(I)=I$ (i.e. $I$ is invariant) or $I^{\prime}=S(I)$ is invariant (being $I^{\prime}=T\left(I^{\prime}\right)$ ).

\section{Proof of Proposition 2.}

Bischi et al. 2000 demonstrated that to each n-cycle of $\phi^{2}:\left\{x_{1}, \ldots, x_{n}\right\}$ there corresponds a conjugate $n$-cycle of given by: $\left\{y_{1}, \ldots, y_{n}\right\}=\left\{\phi\left(x_{1}\right), \ldots, \phi\left(x_{n}\right)\right\}$ and if we consider all the periodic points of $\phi^{2}$ (and their conjugates) then the Cartesian product $\left\{x_{1}, \ldots, x_{n}\right\} \times\left\{y_{1}, \ldots, y_{n}\right\}$ gives all the periodic points of the $\operatorname{map} T$.

\section{Proof of Proposition 3.}

Let $\left\{x_{1}, x_{2}, \ldots, x_{n}\right\}$ be a cycle of $\phi(x)$ of first period $n\left(x_{i+1}=\phi\left(x_{i}\right)\right.$ and $\phi^{n}\left(x_{i}\right)=x_{i}$ for $\left.i=1, \ldots, n\right)$, and consider the points of the Cartesian product $\left\{x_{1}, \ldots, x_{n}\right\} \times\left\{x_{1}, \ldots, x_{n}\right\}$. Then one can immediately compute the iterates by $T$ which are as follows:

$$
T^{k}\left(x_{i}, x_{j}\right)= \begin{cases}\left(\phi^{k}\left(x_{j}\right), \phi^{k}\left(x_{i}\right)\right) & \text { if } k \text { is odd } \\ \left(\phi^{k}\left(x_{i}\right), \phi^{k}\left(x_{j}\right)\right) & \text { if } k \text { is even }\end{cases}
$$

If $i=j$ we have a point on $\Delta$ and thus the first integer giving a cycle is $k=n$ (and we get the $n$-cycle on $\Delta$ ), while for $i \neq j$ we have a point $\left(x_{i}, x_{j}\right)$ external to $\Delta$, and the first integer giving a cycle depends on the period $n$. If $n$ is odd then the first integer giving a periodic point in (21) is $k=2 n$ so that $\left(x_{i}, x_{j}\right)$ belongs to a cycle of $T$ external to $\Delta$ of first period $2 n$. Such distinct cycles must equal $\left(n^{2}-n\right) /(2 n)=(n-1) / 2$ in number. If $n$ is even, then the first integer giving a periodic point in $(21)$ is $k=n$ so that $\left(x_{i}, x_{j}\right)$ is certainly periodic of period $n$, external to $\Delta$, and at most $\left(n^{2}-n\right) / n=(n-1)$ distinct cycles of period $n$ can exist. However, the prime period may be less than $n$. This happens only when $n$ is even and $n / 2$ is odd, and the periodic points belonging to two distinct cycles of period $n / 2$ are $\left(x_{i}, x_{i+n / 2}\right)$ and $\left(x_{i+n / 2}, x_{i}\right)$.

\section{Proof of Proposition 4.}

Consider the case in which two or more cycles of $\phi(x)$, of any pair of periods, coexist. Without loss of generality, let us consider a cycle of period $n$, $n \geq 1$, say $\left\{x_{1}, \ldots, x_{n}\right\}$, and a cycle of period $m, m \geq 1$, say $\left\{y_{1}, \ldots, y_{m}\right\}$. Then it is clear (from Proposition 3) that $n \times n$ points of the type $\left(x_{i}, x_{j}\right)$ belong to singly-generated cycles of $T$, and as well $m \times m$ points of the type $\left(y_{i}, y_{j}\right)$ belong to singly-generated cycles of $T$, but in the Cartesian product $\left\{x_{1}, \ldots, x_{n}, y_{1}, \ldots, y_{m}\right\} \times\left\{x_{1}, \ldots, x_{n}, y_{1}, \ldots, y_{m}\right\}$ we also have other periodic points. Such points belong to cycles which we shall call doubly-generated, because each point of the cycles has the coordinates belonging to two different cycles of $\phi(x)$, so that their existence for the map $T$ is a direct consequence of the existence of 
a pair of cycles of $\phi(x)$. To see this let us consider the iterates by $T$ which are as follows:

$$
T^{k}\left(x_{i}, y_{j}\right)= \begin{cases}\left(\phi^{k}\left(y_{j}\right), \phi^{k}\left(x_{i}\right)\right) & \text { if } k \text { is odd } \\ \left(\phi^{k}\left(x_{i}\right), \phi^{k}\left(y_{j}\right)\right) & \text { if } k \text { is even }\end{cases}
$$

and let us define

$$
L=\operatorname{lcm}(n, m)
$$

where "lcm" stands for "least common multiple". It is clear that when $L$ is odd (which can occur only when both $n$ and $m$ are odd), then the least integer giving a periodic point in (22) is $k=2 L$, and we get a cycle of $T$ of period $2 L$. Such cycles may be $2(n \cdot m) /(2 L)=n \cdot m / L$ in number. When $L$ is even (which occurs when $n$ or/and $m$ are even), then the least integer giving a periodic point in (22) is $k=L$, so that we get a cycle of $T$ of period $L$, and such cycles may be in number $2(n \cdot m) / L$.

Proof of Proposition 5. It is immediate.

\section{Proof of Proposition 6.}

For $A \in \Delta$ we have $P_{x}^{(k)}(A)=P_{y}^{(k)}(A)=\frac{1}{2} I P^{(k)}(A)$ for each $k>0$, and from $I P^{(n)}(A)=n-2 \sum_{i=1}^{n} C_{x_{i}}$ we have $I P^{(p)}(A)=(n-1) n-(n-1) 2 \sum_{i=1}^{n} C_{x_{i}}$.

Now we know (from Proposition 1 ) that the periodic points external to the diagonal are symmetric with respect to $\Delta$, so that we have $p / 2=n(n-1) / 2$ pairs of points external to $\Delta$, and for each pair, say $B=\left(x_{i}, x_{j}\right)$ and $B^{\prime}=\left(x_{j}, x_{i}\right)$, we have $P_{x}(B)+P_{x}\left(B^{\prime}\right)=P_{y}\left(B^{\prime}\right)+P_{y}(B)=1-\left(C_{x_{i}}+C_{x_{j}}\right)$, so that, summing up for all pairs and taking in account that we have only $n$ distinct values, we obtain $P_{x}($ total $)=P_{y}($ total $)=\frac{n(n-1)}{2}-\frac{(n-1)}{2}\left(\sum_{i=1}^{n} C_{x_{i}}+\sum_{j=1}^{n} C_{x_{j}}\right)=$ $\frac{n(n-1)}{2}-(n-1) \sum_{i=1}^{n} C_{x_{i}}$ for the sum of the total profits of the singly generated periodic points. As the total profit is the sum of the two values, we get the same as $I P^{(p)}(A)$.

For $A \in \Delta$ we have $P_{x}^{(k)}(A)=P_{y}^{(k)}(A)=\frac{1}{2} I P^{(k)}(A)$ for each $k>0$, and from $I P^{(n)}(A)=n-2 \sum_{i=1}^{n} C_{x_{i}}$ we have $I P^{(p)}(A)=(n-1) n-(n-1) 2 \sum_{i=1}^{n} C_{x_{i}}$.

Now we know (from Proposition 1 ) that the periodic points external to the diagonal are symmetric with respect to $\Delta$, so that we have $p / 2=n(n-1) / 2$ pairs of points external to $\Delta$, and for each pair, say $B=\left(x_{i}, x_{j}\right)$ and $B^{\prime}=\left(x_{j}, x_{i}\right)$, we have $P_{x}(B)+P_{x}\left(B^{\prime}\right)=P_{y}\left(B^{\prime}\right)+P_{y}(B)=1-\left(C_{x_{i}}+C_{x_{j}}\right)$, so that, summing up for all pairs, and taking in account that we have only $n$ distinct values, we obtain $P_{x}($ total $)=P_{y}($ total $)=\frac{n(n-1)}{2}-\frac{(n-1)}{2}\left(\sum_{i=1}^{n} C_{x_{i}}+\sum_{j=1}^{n} C_{x_{j}}\right)=$ $\frac{n(n-1)}{2}-(n-1) \sum_{i=1}^{n} C_{x_{i}}$ for the sum of the total profits of the singly generated periodic points. As the total profit is the sum of the two values, we get the same as $I P^{(p)}(A)$.

Moreover, whichever is the period $\mathrm{n}$ (odd or even), from the explicit formulas written above we have also obtained that $P_{x}($ total $)=P_{y}($ total $)=\frac{n(n-1)}{2}-(n-$ 1) $\sum_{i=1}^{n} C_{x_{i}}=\frac{1}{2} I P^{(p)}(A)$, which completes the proof. 


\section{References}

Agiza, H.N., 1999, On the Analysis of Stability, Bifurcations, Chaos and Chaos Control of Kopel Map, Chaos, Solitons \& Fractals 10(11), 1909-1916.

Agiza, H.N., G.I. Bischi and M. Kopel, 1999, Multistability in a dynamic Cournot Game with Three Oligopolysts, Mathematics and Computers in Simulation, 51, 63-90.

Agliari, A., L. Gardini and T. Puu, 2000, The dynamics of a triopoly Cournot game, Chaos, Solitons \& Fractals, 11, 2531-2560.

Agliari, A., L. Gardini and T. Puu, 2006, Global Bifurcations in Duopoly when the Cournot Point is Destabilized via a Subcritical Neimark Bifurcation, International Game Theory Review, 8(1), 1-20.

Al-Nowaihi, A. and P.L. Levine, 1985, The Stability of the Cournot Oligopoly Model: A Reassessment, Journal of Economic Theory, 35, 307-321.

Avrutin, V. and M. Schanz, 2006, Multi-parametric bifurcations in a scalar piecewise-linear map, Nonlinearity, 19, 531-552.

Avrutin, V., M. Schanz and S. Banerjee, 2006, Multi-parametric bifurcations in a piecewise-linear discontinuous map, Nonlinearity, 19, 1875-1906.

Banerjee, S., M.S. Karthik, G. Yuan and J.A. Yorke, 2000a, Bifurcations in One-Dimensional Piecewise Smooth Maps - Theory and Applications in Switching Circuits, IEEE Trans. Circuits Syst.-I: Fund. Theory Appl. 47(3), 389-394.

Banerjee, S., P. Ranjan, C. Grebogi, 2000b, Bifurcations in Two-Dimensional Piecewise Smooth Maps - Theory and Applications in Switching Circuits, IEEE Trans. Circuits Syst.-I: Fund. Theory Appl. 47(5), 633-643.

Bischi, G.I. and A. Naimzada, 1999, Global Analysis of a Duopoly Game with Bounded rationality, Advances in Dynamic Games and Applications, 5, 361-385.

Bischi, G.I., L.Gardini and C.Mammana, 2000, Multistability and cyclic attractors in duopoly games, Chaos, Solitons \& Fractals, 11, 543-564.

Bischi, G.I. and M. Kopel, 2001, Equilibrium Selection in a Nonlinear Duopoly Game with Adaptive Expectations, Journal of Economic Behavior and Organization, 46, 73-100.

Bonanno, G., 1988, Oligopoly Equilibria when firms have local knowledge of Demand, International Economic Review, 29 (1), 45-55.

Dana, R.-A. and L. Montrucchio, 1986, Dynamic Complexity in Duopoly Games, Journal of Economic Theory, 40, 40-56.

Di Bernardo M., M.I. Feigen, S.J. Hogan and M.E. Homer, 1999, Local analysis of C-bifurcations in n-dimensional piecewise smooth dynamical systems, Chaos, Solitons \& Fractals 10(11), 1881-1908.

Fisher, F.M., 1961, The stability of the Cournot oligopoly solution: The effects of speeds of adjustment and increasing marginal costs, Review of Economic Studies, 28, 125-135.

Furth, D., 1986, Stability and Instability in Oligopoly, Journal of Economic Theory, 40, 197-228. 
Gallegati, M., L. Gardini, T. Puu and I. Sushko, 2003, Hicks Trade Cycle Revisited: Cycles and Bifurcations, Mathematics and Computers in Simulation, 63, 505-527.

Halse, C., M. Homer and M. di Bernardo, 2003, C-bifurcations and periodadding in one-dimensional piecewise-smooth maps, Chaos, Solitons \& Fractals 18, 953-976.

Hommes, C.H., 1991, Chaotic Dynamics in Economic Models: Some Simple Case Studies, Thesis University of Groningen, Wolters-Noordhoff Groningen

Hommes, C.H. and E. Nusse, 1991, Period three to period two bifurcations for piecewise linear models, Journal of Economics 54(2), 157-169.

Hommes, C.H., E. Nusse and A. Simonovits, 1995, Cycles and chaos in a socialist economy, Journal of Economic Dynamic and Control, 19, 155-179.

Hommes, C.H. 1995, A reconsideration of Hick's non-linear trade cycle model, Structural Change and Economic Dynamics 6, 435-459.

Kopel, M. 1996, Simple and Complex adjustment dynamics in Cournot Duopoly Models, Chaos, Solitons, \& Fractals (7), 2031-2048.

Leonov N.N., 1959, Map of the line onto itself, Radiofisica 3(3), pp. 942-956.

Leonov N.N., 1962, Discontinuous map of the straight line, Dohk. Ahad. Nauk. SSSR. 143(5) pp. 1038-1041.

McManus, M. and R.E. Quandt, 1961, Comments on the stability of the Cournot oligopoly model, Review of Economic Studies, 28, 136-139.

Maistrenko, Y.L., V.L. Maistrenko and L.O. Chua, 1993, Cycles of chaotic intervals in a time-delayed Chua's circuit, International Journal of Bifurcation and Chaos 3(6), 1557-1572.

Maistrenko, Y.L., V.L. Maistrenko, S.I. Vikul and L.O. Chua, 1995, Bifurcations of attracting cycles from time-delayed Chua's circuit, International Journal of Bifurcation and Chaos 5(3), 653-671.

Maistrenko, Y.L., V.L. Maistrenko and S.I. Vikul, 1998, On period-adding sequences of attracting cycles in piecewise linear maps, Chaos, Solitons \& Fractals $9(1), 67-75$.

Mira C., 1978, Sur les structure des bifurcations des diffeomorphisme du cercle, C.R.Acad. Sc. Paris, 287, Series A, pp. 883-886.

Mira C., 1987, Chaotic dynamics (World Scientific, Singapore).

Mira C., L. Gardini, A. Barugola and J.C. Cathala, 1996, Chaotic dynamics in two-dimensional noninvertible maps (World Scientific, Singapore).

Nusse, H.E. and J.A. Yorke, 1992, Border-collision bifurcations including period two to period three for piecewise smooth systems, Physica D 57, 39-57.

Nusse, H.E. and J.A Yorke, 1995, Border-collision bifurcation for piecewise smooth one-dimensional maps, International Journal of Bifurcation and Chaos 5(1), 189-207.

Okuguchi, K., 1976, Expectations and Stability in Olygopoly Models (Springer Verlag, N.Y.).

Palander, T.F., 1939, Konkurrens och marknadsjämvikt vid duopol och oligopol, Ekonomisk Tidskrift 41, 124-145, and 222-250.

Puu, T., 1991, Chaos in Duopoly Pricing, Chaos, Solitons \& Fractals (1), $573-581$. 
Puu, T., 1996, Complex Dynamics with Three Olygopolists, Chaos, Solitons, \& Fractals 7(12), 2075-2081.

Puu, T., 2005, Layout of a New Industry: From Oligopoly to Competition, Pure Mathematics and Applications, 16:475-492.

Puu, T., 2007a, On the Stability of Cournot Equilibrium when the Number of Competitors Increases, Journal of Economic Behavior and Organization (to appear).

Puu, T., 2007b, The Road from Imperfect to Perfect Competition (submitted).

Puu, T., L. Gardini and I. Sushko, 2002, Cournot duopoly with kinked linear demand according to Palander and Wald, in T. Puu and I. Sushko (Eds.) Oligopoly and Complex Dynamics: Tools and Models 111-146 (Springer-Verlag, N.Y.)

Puu, T., L. Gardini,and I. Sushko, 2005, A Hicksian Multiplier-Accelerator Model with Floor Determined by Capital Stock, Journal of Economic Behavior and Organization, 56, 331-348.

Puu, T. and I. Sushko, 2002, Oligopoly Dynamics, Models and Tools (Springer Verlag, N.Y.)

Puu, T. and I. Sushko, 2006, Business Cycle Dynamics, Models and Tools (Springer Verlag, N.Y.)

Puu, T., L. Gardini and I. Sushko, 2005, A Hicksian Multiplier-Accelerator Model with Floor Determined by Capital Stock, Journal of Economic Behavior and Organization, 56, 331-348.

Sushko, I., L. Gardini and T. Puu, 2004, Tongues of periodicity in a family of two-dimensional discontinuous maps of real Möbius type, Chaos, Solitons \& Fractals 21, 403-412.

Sushko, I, T. Puu and L. Gardini, 2003, The Hicksian floor-roof model for two regions linked by interregional trade. Chaos, Solitons \& Fractals (18), 593612 .

Sushko, I., A. Agliari and L. Gardini, Bistability and border-collision bifurcations for a family of unimodal piecewise smooth maps, Discrete and Continuous Dynamical Systems, Serie B, 5(3) (2005), 881-897.

Sushko, I, A. Agliari and L. Gardini, 2006, Bifurcation Structure of Parameter Plane for a Family of Unimodal Piecewise Smooth Maps: Border-Collision Bifurcation Curves, Chaos, Solitons \& Fractals, 29(3), 756-770.

Theocharis, R.D., 1960, On the stability of the Cournot solution on the Oligopoly Problem, Review of Economic Studies, 27, 133-134.

Zhanybai, T., Zhusubaliyev and E. Mosekilde, 2003, Bifurcations and Chaos in Piecewise-Smooth Dynamical Systems, Singapore (World Scientific, Singapore) 